\title{
OPEN Salicylic acid is a key player of Arabidopsis autophagy mutant susceptibility to the necrotrophic bacterium Dickeya dadantii
}

\author{
Martine Rigault, Sylvie Citerne, Céline Masclaux-Daubresse \& Alia Dellagi ${ }^{\bowtie}$
}

Autophagy is a ubiquitous vesicular process for protein and organelle recycling in eukaryotes. In plant, autophagy is reported to play pivotal roles in nutrient recycling, adaptation to biotic and abiotic stresses. The role of autophagy in plant immunity remains poorly understood. Several reports showed enhanced susceptibility of different Arabidopsis autophagy mutants ( $\mathrm{atg}$ ) to necrotrophic fungal pathogens. Interaction of necrotrophic bacterial pathogens with autophagy is overlooked. We then investigated such interaction by inoculating the necrotrophic enterobacterium Dickeya dadantii in leaves of the atg 2 and atg 5 mutants and an ATG8a overexpressing line. Overexpressing ATG8a enhances plant tolerance to $D$. dadantii. While atg 5 mutant displayed similar susceptibility to the WT, the atg2 mutant exhibited accelerated leaf senescence and enhanced susceptibility upon infection. Both phenotypes were reversed when the sid2 mutation, abolishing SA signaling, was introduced in the atg 2 mutant. High levels of SA signaling in atg 2 mutant resulted in repression of the jasmonic acid (JA) defense pathway known to limit $D$. dadantii progression in $A$. thaliana. We provide evidence that in atg2 mutant, the disturbed hormonal balance leading to higher SA signaling is the main factor causing increased susceptibility to the $D$. dadantii necrotroph by repressing the JA pathway and accelerating developmental senescence.

Being sessile organisms, plants cannot escape the multitude of stresses they are exposed to. They can be attacked by microbial pathogens and have the ability to protect themselves by the deployment of complex defense mechanisms. Defense-related responses involve protein phosphorylation, accumulation of reactive oxygen species (ROS), ionic fluxes and biosynthesis of phytohormones leading to transcriptional activation of genes coding for enzymes involved in the synthesis of antimicrobial compounds such as phytoalexins or pathogenesis related (PR) proteins ${ }^{1-6}$. To activate these defenses, plants are equipped with receptors that can detect different types of molecules either derived from the pathogen or derived from their own tissues ${ }^{7-9}$. Hormones are strongly involved in plant signaling during pathogen attack ${ }^{10-13}$. Depending on the lifestyle of the pathogen, different hormone pathways can be more or less active ${ }^{14,15}$. For instance, plant defense against necrotrophs or insects is generally mediated by the jasmonic acid (JA) pathway, while defense against biotrophic pathogens is mediated by the salicylic acid (SA) pathway. Several reports indicate the existence of cross-talks between those defense signaling pathways ${ }^{11,12,16}$. An antagonism was generally described between SA dependent defenses and JA/ET dependent defenses ${ }^{17-20}$. The fact that the activation of one of them represses the other suggests that plants are able to prioritize the signaling pathway they activate upon infection ${ }^{16}$. Interestingly, synergism between SA and JA pathways was also described ${ }^{21}$.

Autophagy is an evolutionary conserved process involved in the degradation of unwanted cell material ${ }^{22-24}$. Although the main mechanism by which autophagy contributes to cell homeostasis is thought te be the degradation of cytoplasmic components, the involvement of autophagy in protein secretion is another emerging mechanism ${ }^{25,26}$. Autophagy consists in the formation of a double membrane vesicle, named autophagosome, that forms arround and encloses the cargoes to be degraded ${ }^{27-29}$. When cargoes are captured, autophagososmes drive them towards the lytic vacuole for degradation ${ }^{27-29}$.

Autophagy is tightly controlled. Autophagosomes likely target specific cargoes through their interaction with the ATG8 proteins that are lipidated and anchored to autophagosome membranes. The autophagy proteins (ATG) involved in this complex machinery were first identified in yeast ${ }^{30}$. Most of their orthologs were found in 
mammals and in plants. In plant, autophagy process was found to be compromised in all the atg mutants defective in the single ATG genes ${ }^{23,24,31}$. The ATG8 protein is encoded by nine genes in Arabidopsis and although the ATG8 protein is a key player of the autophagy core machinery, no phenotype had been reported so far for the different atg8 single mutants isolated in Arabidopsis, possibly due to functional redundancies. The conjugation of ATG8 to phosphatidyl-ethanolamine (PE) relies on a complex conjugation system that involves the ATG5 protein. The ATG2 protein is involved in lipid recruitment for autophagosome membrane elongation. Both ATG5 and ATG2 are encoded by single genes and their mutants display strong senescence and limited-growth phenotypes although phenotypes are more severe in $\operatorname{atg} 2$ than in $\operatorname{atg} 5^{32-35}$. Autophagy is post-transcriptionally regulated by the TOR protein kinase (Target of Rapamycin ${ }^{36-38}$ ). Autophagy genes are up-regulated under stress conditions, amongst which plant infection by pathogens ${ }^{39,40}$.

In plants, recent studies show that autophagy is involved in plant pathogen interactions and the involvement of autophagy machinery in disease/resistance is highly dependent on the pathosystems as well as on the plant physiological status ${ }^{41-44}$. The fine tuning of the cell death related to the hypersensitive response (HR), which is a strong resistance mechanism, is altered in autophagy mutants ${ }^{34,45}$. In addition, several studies show that compromising autophagy results in an enhanced susceptibility to necrotrophic fungi ${ }^{46-48}$. However it remains unclear if alteration in plant immunity is directly due to the lack of autophagy degradation process or results from indirect effects of autophagy alteration.

Dickeya dadantii is a necrotrophic plant pathogenic bacterium that causes soft rot disease on a large host range of crops and is able to infect Arabidopsis thaliana ${ }^{49,50}$. As is is the case of many other necrotrophs, D. dadantii produces large amounts of plant cell wall degrading enzymes that cause soft rots of plant tissues. Several lines of defense allow the plants to limit infection by $D$. dadantii including the production of ROS via the disturbance of iron homeostasis and by the membrane located NADPH oxidase ${ }^{50-53}$. Several lines of evidence show that $D$. dadanti triggers JA defense pathway ${ }^{50,53}$.

The role of autophagy in plant tolerance to pathogens was mainly documented regarding the cell death hypersensitive response to bacteria such as Pseudomonas and in response to necrotrophic fungi ${ }^{44,48}$. So far, there is no report dealing with necrotrophic bacteria and autophagy. Here, we show that the defect of Arabidopsis autophagy mutants' tolerance to the bacterial necrotroph $D$. dadantii is not linked to the autophagy activity per se, but is an indirect effect of impaired fine tuning of SA defense signaling.

\section{Materials and methods}

Plant material. Wild type accession of Arabidopsis thaliana Col-0 was obtained from Versailles Arabidopsis Stock Center (INRA Versailles France, http://publiclines.versailles.inra.fr/). The autophagy mutants atg2 (SALK_076727), atg5 (SAIL_129B07), atg2.sid2, atg5.sid2 were obtained from Yoshimoto et al. and MasclauxDaubresse et al. ${ }^{54}$. The sid2 mutant was kindly provided by Pr. JP Métraux. The $p U B I:: A T G 8 a:: G F P$ overexpressor Arabidopsis line was obtained from Chen et al. ${ }^{55}$.

Bacterial inoculation and quantification of disease severity. Inoculation experiments were performed with the $D$. dadantii 3937 strain as described in $^{56}$. Bacteria were grown in Luria-Bertani medium. Plants used for RNA extraction were inoculated by leaf infiltration using a syringe without a needle with a bacterial suspension at $1 \times 10^{8}$ Colony Forming Unit/mL (CFU) made up in $10 \mathrm{mM} \mathrm{MgSO}$, mock inoculated controls consisted of leaves infiltrated with $10 \mathrm{mM} \mathrm{MgSO}_{4}$. Plants used for symptom scoring were inoculated by making a small hole with a needle in the leaf limb, and then spotting $5 \mu \mathrm{L}$ of a bacterial suspension at a density of $1 \times 10^{8} \mathrm{CFU} / \mathrm{mL}$ made up in $50 \mathrm{mM}$ potassium phosphate buffer $(\mathrm{pH} 7)$ on the top of the hole. Symptom severity scoring was performed according to the 0 to 5 severity scale described in Rigault et al. ${ }^{56}$ and indicated in Supplementary Figure 1. Each symptom severity on inoculated leaves is scored then an average and a standard deviation are calculated.

Monitoring plant gene expression by qRT-PCR. RNA extractions and RT-qPCR were performed as described in Verly et al. and Aznar et al. ${ }^{57,58}$ Leaves were harvested $24 \mathrm{~h}$ post inoculation (H p.i.) and then frozen in liquid nitrogen. Total RNAs were purified with TRIzol reagent (Invitrogen, Carlsbad, CA, USA) according to the manufacturer's instructions. The total RNA concentration was determined using a NanoDrop ND-1000 spectrophotometer (NanoDropTechnologies Inc., Wilmington, DE, USA). RNA samples were treated with Turbo DNaseI (Ambion, Saint-Aubin, France) RNase-free to remove any DNA contamination. A total of $1 \mu \mathrm{g}$ of DNase-treated RNA was reverse transcribed using the High Capacity cDNA Reverse Transcription Kit and 50 ng of random hexamers following the supplier's instructions. One microlitre of the 1:10 diluted cDNA was subjected to real-time quantitative PCR using SYBR Green PCR Mastermix (Applied Biosystems, Foster City, CA, USA) and gene-specific primers designed to amplify 100-150-bp fragments from each gene of interest and the reference genes APT and Clathrin. Primer sequences used for qRT-PCR are indicated in Supplementary Table 1.

Salicylic acid quantification. For each sample, $2 \mathrm{mg}$ of dry powder were extracted with $0.8 \mathrm{~mL}$ of acetone/ water/acetic acid (80/19/1 v:v:v). Salicylic acid stable labelled isotope used as internal standard was prepared as described in Le Roux et al..$^{59} .1 \mathrm{ng}$ of standard was added to each sample. The extract was vigorously shaken for $1 \mathrm{~min}$, sonicated for $1 \mathrm{~min}$ at $25 \mathrm{~Hz}$, shaken for $10 \mathrm{~min}$ at $10^{\circ} \mathrm{C}$ in a Thermomixer (EPPENDORF, and then centrifuged at $8000 \mathrm{~g}, 10^{\circ} \mathrm{C}$ for $10 \mathrm{~min}$ ). The supernatants were collected, and the pellets were re-extracted twice with $0.4 \mathrm{~mL}$ of the same extraction solution, then vigorously shaken $(1 \mathrm{~min})$ and sonicated $(1 \mathrm{~min} ; 25 \mathrm{~Hz})$. After the centrifugations, the three supernatants were pooled and dried (final volume $1.6 \mathrm{~mL}$ ).

Each dry extract was dissolved in $100 \mu \mathrm{L}$ of acetonitrile/water $(50 / 50 \mathrm{v} / \mathrm{v})$, filtered, and analyzed using a Waters Acquity ultra performance liquid chromatograph coupled to a Waters Xevo Triple quadrupole mass 


\begin{tabular}{|c|c|c|c|c|}
\hline Necrotrophic pathogen & Construct name & Mutant/ox & Phenotype & Ref \\
\hline B. cinerea & $\operatorname{atg} 5-1$ & Mutant & Enhanced susceptibility & 47 \\
\hline B. cinerea & $\operatorname{atg} 7-3$ & Mutant & Enhanced susceptibility & 47 \\
\hline B. cinerea & $\operatorname{atg} 7-2$ & Mutant & Enhanced susceptibility & 47 \\
\hline B. cinerea & atg18a-1 & Mutant & Enhanced susceptibility & 47 \\
\hline B. cinerea & ATG 18a-RNAi & Mutant & Enhanced susceptibility & 47 \\
\hline A. brassicicola & $\operatorname{atg} 5-1$ & Mutant & Enhanced susceptibility & 47 \\
\hline A. brassicicola & $\operatorname{atg} 7-3$ & Mutant & Enhanced susceptibility & 47 \\
\hline A. brassicicola & $\operatorname{atg} 7-2$ & Mutant & Enhanced susceptibility & 47 \\
\hline A. brassicicola & atg18a-1 & Mutant & Enhanced susceptibility & 47 \\
\hline A. brassicicola & ATG 18a-RNAi & Mutant & Enhanced susceptibility & 47 \\
\hline A. brassicicola & atg5 & Mutant & Enhanced susceptibility & 46 \\
\hline A. brassicicola & $\operatorname{atg} 10$ & Mutant & Enhanced susceptibility & 46 \\
\hline A. brassicicola & atg18a-1 & Mutant & Enhanced susceptibility & 46 \\
\hline A. brassicicola & atg18a-2 & Mutant & Enhanced susceptibility & 46 \\
\hline A. brassicicola & $\operatorname{atg} 5 / A T G 5$ & Complemented mutant & Restored WT susceptibility & 46 \\
\hline A. brassicicola & atg10/ATG10 & Complemented mutant & Restored WT susceptibility & 46 \\
\hline B. cinerea & $\operatorname{atg} 2$ & Mutant & No effect & 65 \\
\hline Sclerotinia & $\operatorname{atg} 7$ & Mutant & No effect & 66 \\
\hline Sclerotinia & atg8e & Mutant & No effect & 66 \\
\hline Sclerotinia & $\operatorname{atg} 12$ & Mutant & No effect & 66 \\
\hline B. cinerea & $\operatorname{atg} 5$ & Mutant & Enhanced susceptibility & 74 \\
\hline B. cinerea & $\operatorname{atg} 7$ & Mutant & Enhanced susceptibility & 74 \\
\hline Plectosphaerella cucumerina & $\operatorname{atg} 5$ & Mutant & Enhanced susceptibility & 46 \\
\hline Plectosphaerella cucumerina & $\operatorname{atg} 10$ & Mutant & Enhanced susceptibility & 46 \\
\hline Plectosphaerella cucumerina & atg5/ATG5 & Complemented mutant & Restored WT susceptibility & 46 \\
\hline Plectosphaerella cucumerina & atg10/ATG10 & Complemented mutant & Restored WT susceptibility & 46 \\
\hline
\end{tabular}

Table 1. List of studies about autophagy involvement in disease cause by necrotrophic fungi on Arabidopsis.

spectrometer TQS (UPLC-ESI-MS/MS). The compounds were separated on a reverse-phase column (Uptisphere C18 UP3HDO, $100 * 2.1 \mathrm{~mm}{ }^{*} 3 \mu \mathrm{m}$ particle size; Interchim, France) using a flow rate of $0.4 \mathrm{~mL} \mathrm{~min}^{-1}$ and a binary gradient: $(\mathrm{A})$ acetic acid $0.1 \%$ in water $(\mathrm{v} / \mathrm{v})$ and $(\mathrm{B})$ acetonitrile with $0.1 \%$ acetic acid, the column temperature was $40{ }^{\circ} \mathrm{C}$, we used the following binary gradient (time, $\left.\% \mathrm{~A}\right)$ : (0 min, $\left.98 \%\right),(3 \mathrm{~min}, 70 \%),(7.5 \mathrm{~min}$, 50\%), (8.5 $\mathrm{min}, 5 \%$ ), (9.6 $\mathrm{min}, 0 \%$ ), (13.2 $\mathrm{min}, 98 \%)$, (15.7 min, 98\%).

Mass spectrometry was conducted in electrospray and Multiple Reaction Monitoring scanning mode (MRM mode), in negative ion mode. Relevant instrumental parameters were set as follows: capillary $1.5 \mathrm{kV}$ (negative mode), source block and desolvation gas temperatures $130{ }^{\circ} \mathrm{C}$ and $500{ }^{\circ} \mathrm{C}$, respectively. Nitrogen was used to assist the cone and desolvation $\left(150 \mathrm{~L} \mathrm{~h}^{-1}\right.$ and $800 \mathrm{~L} \mathrm{~h}^{-1}$, respectively), argon was used as the collision gas at a

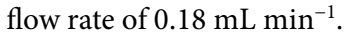

\section{Results}

Arabidopsis tolerance to the bacterial necrotroph $D$. dadantii is altered in autophagy mutants but does not necessitate autophagy activity. The role of autophagy in Arabidopsis tolerance to necrotrophic phytopathogens was only reported for fungal plant pathogens (Table 1), using atg mutants or transgenic lines over-expressing $A T G$ genes. Consistent data were obtained in all reports showing that atg mutants display increased susceptibility to necrotrophic fungi. It is not the case for biotrophs and hemibiotrophs for which the role of plant autophagy depends on the pathosystem ${ }^{44,48}$. To know whether autophagy is also involved in Arabidopsis tolerance to bacterial necrotrophs, we addressed the issue with the model bacterial necrotroph Dickeya dadantii ${ }^{60}$.

For this purpose, two different atg mutants, one affected in lipid recruitment for autophagosome formation (atg2) and the other affected in the conjugation system permitting ATG8 lipidation and anchorage to the membrane of autophagosomes (atg5) were inoculated with $D$. dadantii. Wild type Col-0 and the two atg 2 and $\operatorname{atg} 5$ mutants were inoculated by spotting a bacterial suspension on leaves of 6 weeks old plants. The severity of the symptoms was scored based on $0-5$ scale $^{56}$. Severity of the symptoms on atg2 mutant was higher over time than that observed on the WT plants (Fig. 1). Interestingly, the susceptibility level of the atg5 mutant was similar to that of the WT. The absence of difference between atg 5 mutant and WT was confirmed on the two atg5 mutant allele (atg5-1 and $\operatorname{atg} 5-2^{33}$; data not shown). This indicates that only atg 2 was more susceptible than Col-0 to $D$. dadantii while the atg 5 mutant known to display a less severe yellowing phenotype than atg2, was not affected in its susceptibility to $D$. dadantii. Such difference between atg2 and atg5, then raised the question of the role of 

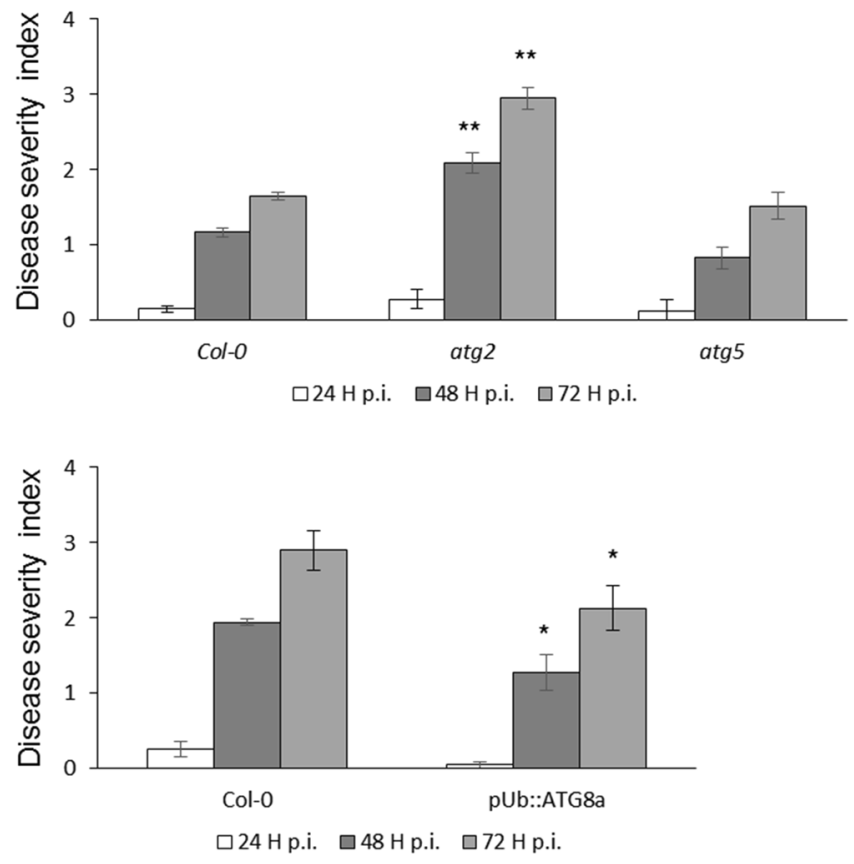

Figure 1. Disease severity on atg mutant and ATG8a overexpressing lines: indicated genotypes were inoculated with $D$. dadantii and disease severity was monitored over 3 days according to the severity scale ${ }^{56}$. Bars are standard errors. $\mathrm{N}=60$ leaves from 20 plants. ${ }^{\star *} \mathrm{p}<0.01,{ }^{*} \mathrm{p}<0.05 t$ test comparing means to the Col- 0 WT. Experiments were performed 3 times with similar results.

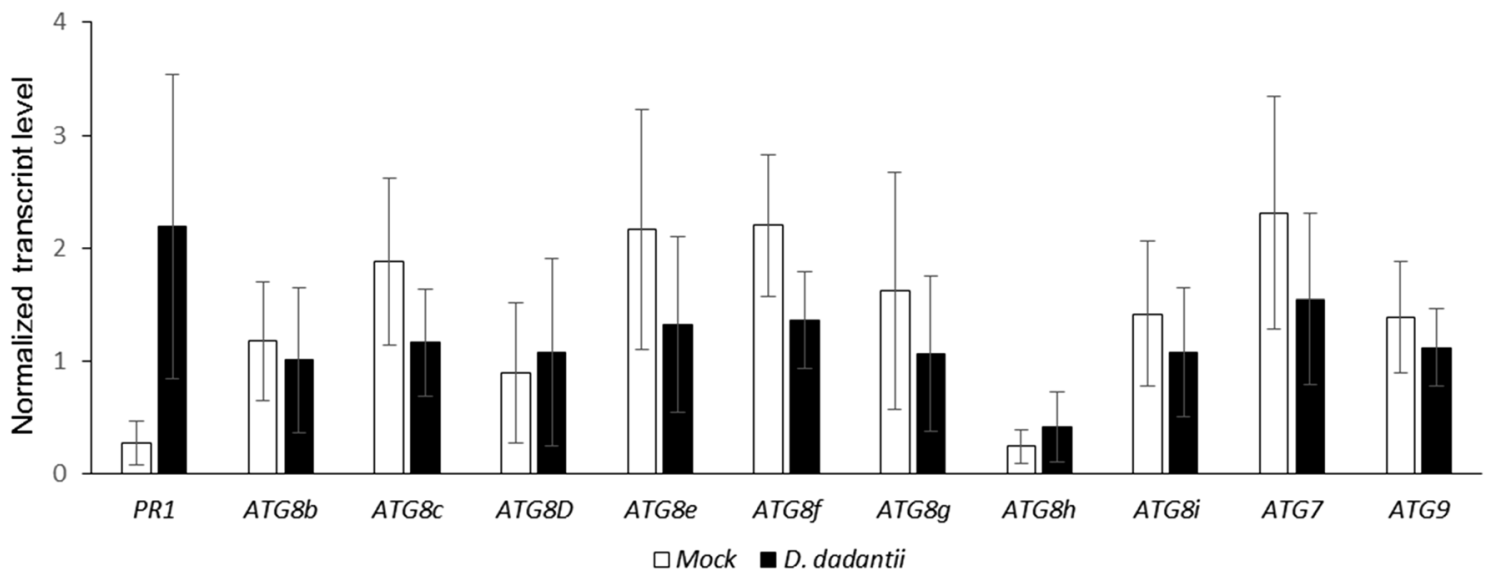

Figure 2. Expression of $A T G$ genes upon $D$. dadantii infection. Plants were infected or mock treated then leaves were harvested $24 \mathrm{~h}$ after treatment. Transcript levels were monitored by qRT-PCR and normalized against the transcripts of the reference genes $A P T$ and Clathrin. Bars are standard deviation, $\mathrm{N}=4$. Experiments were performed 3 times with similar results.

the autophagy machinery in the tolerance to D. dadantii, and the potential link with a senecence status of the mutants.

We then used an Arabidopsis line with enhanced autophagic activity consisting in the overexpression of the ATG8a protein under the control of Ubiquitin promoter ${ }^{55}$. Following $D$. dadantii inoculation, plants overexpressing ATG8a displayed reduced symptom severity compared to the WT suggesting a positive role of autophagy activity on the tolerance of Arabidopsis to D. dadantii (Fig. 1).

Autophagy genes are not up-regulated upon D. dadantii infection. As disease symptoms observed on the different atg lines suggested a link between plant response to $D$. dadantii and autophagy, we monitored $A T G$ gene expression following plant inoculation. RT-qPCR was performed to monitor the transcript level of 10 autophagy genes chosen for their positive response to stress in litterature ${ }^{40}$. None of these genes was found to be upregulated in response to infection (Fig. 2) by contrast with the defense gene marker PR1 that was upregulated 
in the same plants as expected. These data suggest that transcriptional activation of autophagy genes was not required in the response of Arabidopsis to D. dadantii infection.

Salicylic acid dependent susceptibility of atg2 mutant to $D$. dadantii. Our results indicate that functional ATG2 protein is essential to control disease symptoms in Arabidopsis upon D. dadantii infection but that working ATG5 protein and operational autophagy is not essential. Even though autophagy genes are not induced by infection, enhanced constitutive autophagic activity provides positive effect on plant tolerance to D. dadantii. If autophagy machinery was directly involved in Arabidopsis tolerance to the bacterium, then any knock-out mutant affected in autophagy should be more susceptible to the bacterium. Our results suggest that defect in autophagy activity was not the direct cause of Arabidopsis susceptibility to $D$. dadantii. Indirect effects of autophagy defects on plant tolerance to pathogens might be related to hormonal balance. It is well known that autophagy defect triggers hormonal disorders and especially exacerbates SA production and SA signaling, then enhancing fast and spectacular leaf senescence like symptoms ${ }^{41,42}$. Because senescence/yellowing phenotypes observed on the atg 2 mutant is stronger than in the atg 5 mutant, we suspected exacerbation of SA response in atg 2 by comparison with atg 5 and considered the possibility that SA accumulation in atg 2 was the origin of its enhanced susceptibility to $D$. dadantii.

In order to determine whether SA is involved in the increased susceptibility of $\operatorname{atg} 2$ mutant, we monitored the susceptibility of the atg2.sid2 double mutant defective in SA synthesis as the SID2 gene encodes an isochorismate synthase which is involved in SA biosynthesis in Arabidopsis, in particular in response to pathogens ${ }^{61}$. Figure 3A shows the phenotypes of Col-0 wild type, atg2 and atg2.sid2 mutants infected with $D$. dadantii. Both soft rot symptoms and leaf yellowing phenotypes were clearly more severe on atg2 leaves than on Col-0 and atg2.sid2 leaves (Fig. 3). Although atg 2 was early senescing by comparison with Col-0 and atg2.sid2 plants ${ }^{34}$ (Fig. 3A), we observed enhanced chlorosis around the $D$. dadantii inoculation spots which were not present in uninfected plants or before inoculation (data not shown). This suggested that infection accelerated leaf senescence symptoms on atg 2 plants. It can be noticed that disease severity was similar in WT, sid2 and atg2.sid2 double mutant. These data indicate that the higher susceptibility of the atg 2 mutant can be restored to the WT level when SID2 gene is non-functional, suggesting the prominent role of SA in the increased susceptibility of atg2 mutant to $D$. dadantii. This confirms that autophagy degradation pathway is not essential for Arabidopsis tolerance to $D$. dadantii. Nevertheless, higher constitutive autophagic activity artificially enhanced by ATG8a overexpression may help increasing plant tolerance, possibly through positive effects on leaf longevity, meaning negative effect on SA production.

Salicylic acid signaling rather than SA amount is involved in atg 2 mutant enhanced susceptibility to $D$. dadantii. Previous reports indicate that the level of salicylic acid in autophagy mutants is higher than that of $\mathrm{WT}^{54,62}$. To determine the mechanism by which high SA level could lead to enhanced susceptibility, we hypothesized that high amounts of SA could repress the JA signaling pathway which is known to limit $D$. dadantii infection ${ }^{50,57}$. Indeed, it is commonly reported that SA signaling represses JA signaling and that ET and JA signaling are commonly synergistic ${ }^{17-20,63}$.

To further investigate the possibility that SA and JA signaling were modified in atg2 mutants, we determined the status of $S A$ and JA mediated defenses in Col-0, sid2, atg2, atg5, atg2.sid2 and atg5.sid2 plants. For this purpose, we monitored the expression of defense marker genes of the SA (PR1) and the JA (PDF1.2) pathways in infected plants. Controls consisted of plants inoculated with $10 \mathrm{mM} \mathrm{MgSO}_{4}$ and naïve plants that were untreated. The latter naïve plants were used to determine the initial immunity level of plants before inoculation. Transcript levels of both the SA defense marker gene PR1 and of the JA defense marker gene PDF1.2 were increased in WT Col-0 plants following D. dadantii infection compared to controls (Fig. 4). While no PR1 transcript was detected in the sid2 mutant line, higher levels of PDF1.2 transcripts were detected in these plants compared to Col-0 confirming the repressive action of the SA pathway on the JA pathway. Strikingly, in the atg2 mutant, higher levels of PR1 transcripts were detected compared to the WT (10 times higher in atg 2 compared to Col-0 infected plants) and an up-regulation of this level was observed in response to $D$. dadantii compared to the mock inoculated plants. The level of $P R 1$ transcripts in $\operatorname{atg} 5$ mutant was unchanged compared to the WT indicating that SA signaling in the atg5 mutant was not affected. The expression level of PDF1.2 was reduced in the atg 2 mutant compared to that of Col- 0 which is consistent with a high SA signaling. The transcript level of $P R 1$ was undetectable in both atg2.sid2 and atg5.sid2 double mutants, indicating that SA signaling was totally abolished by introducing the sid2 mutation. Interestingly, in naïve atg simple and double mutant plants, the level of PDF1.2 transcripts was reduced compared to that of mock inoculated plants. Our data suggest that mechanical stress triggers JA signaling in atg mutant backgrounds, a process which is repressed by $D$. dadantii infection. To know whether senescence was also involved in this interaction, the transcript level of the senescence marker gene SAG12 was monitored. SAG12 was only expressed in atg2 mutant plants (Fig. 4). Intriguingly, it was down regulated by mock inoculation and up-regulated by infection confirming the accelerated senescing phenotype we observed on atg2 infected plants compared to mock atg2 plants.

To investigate the role of SA content in the response of autophagy mutants to $D$. dadantii, SA content was quantified in naïve plants (Fig. 5). Both atg2 and atg5 mutants accumulate higher SA levels than Col-0 plants. The level of SA was reduced in both double mutants atg2.sid2 and atg5.sid2 relative to single mutants confirming the role of SID2 in SA production in Arabidopsis. 
A

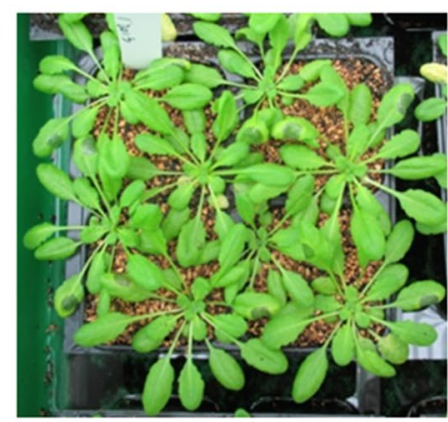

Col-O
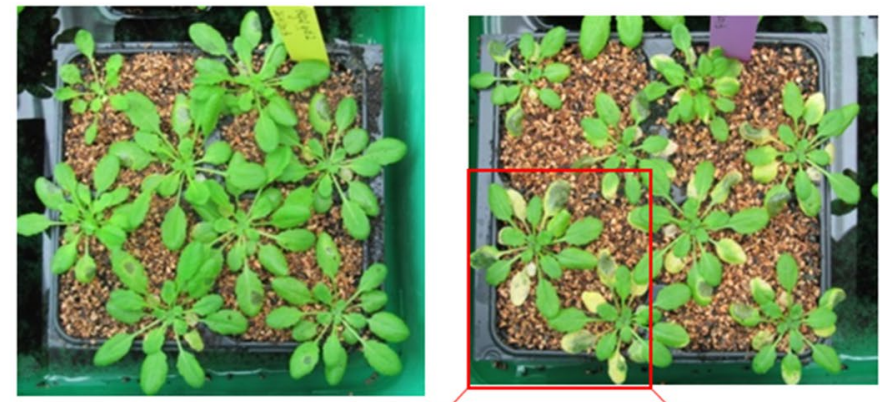

$\operatorname{atg} 2 . s i d 2$

B

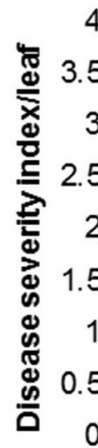

a_3

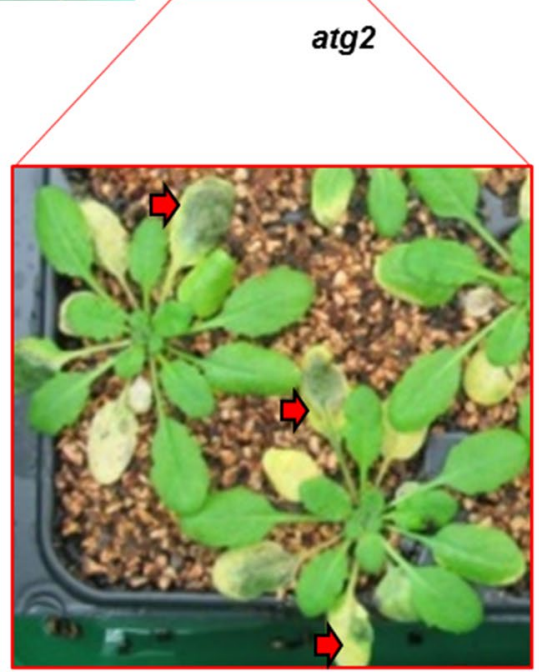

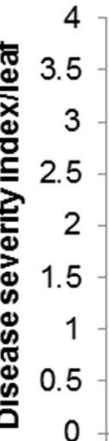
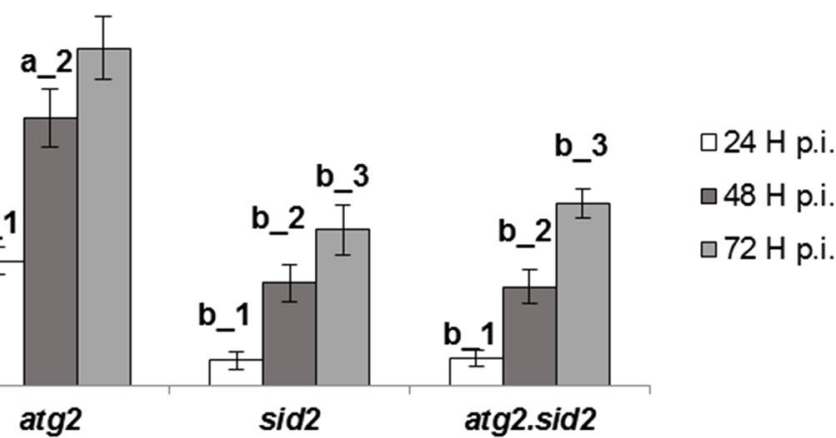

b_3

b_1

b_2
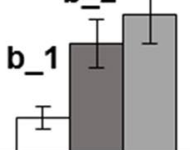

Col-0

Figure 3. Susceptibility of atg2 mutant relies on SA. (A) Pictures of 6 week-old Arabidopsis indicated genotypes inoculated with $D$. dadantii. In the enlargement of atg2 mutant inoculated plants, red arrows indicate yellow zones of early senesce. (B) Symptom severity at indicated time points on indicated genotypes. Bars represent standard deviation. $\mathrm{N}=30$. Experiments were performed three times with similar results. Different letters in the graph indicate statistical significance between genotypes at the same time point (one-way ANOVA with Tuckey's test; $\mathrm{P}<0.05)$.

\section{Discussion}

The mechanisms by which plant autophagy affects immunity are complex and vary depending on the considered pathosystems. In some cases, opposite mechanisms can take place depending on plant age or infectious stage. For example, in young Arabidopsis leaves, autophagy has a pro-death activity enhancing HR mediated cell death ${ }^{45}$ while in older leaves, autophagy seems to counteract cell death ${ }^{34}$. Plant autophagy was shown to counteract the bacterial pathogen Pseudomonas syringae pv. tomato which, surprisingly, secretes an effector able to trigger autophagy ${ }^{64}$.

Several reports indicate that the susceptibility of atg mutants was increased in response to fungal necrotrophic pathogens (Table 1). To determine the effect of atg mutations on Arabidopsis susceptibility to the bacterial necrotroph D. dadantiii, we used an atg2 and atg5 mutants. The ATG2 and ATG5 genes are single genes in Arabidopsis as in many plant species, and are essential for autophagic activity as their mutants cannot form autophagosomes. The atg 2 and atg 5 mutants were previously characterized by many groups amongst which Pr. R. Vierstra and Pr. K. Yoshimoto groups, and display the typical hypersensitivity to abiotic stresses described for many autophagy 

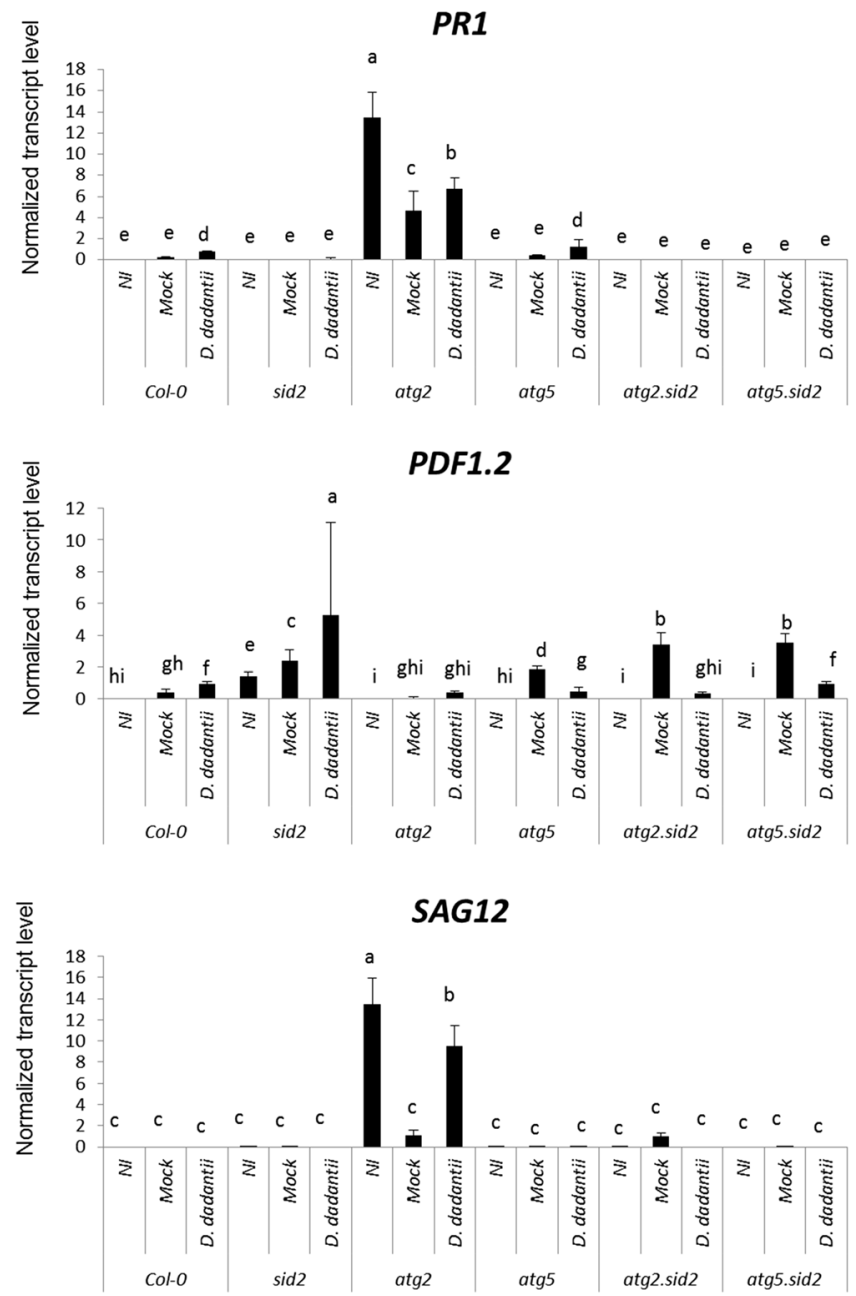

Figure 4. Expression of defense and senescence genes upon $D$. dadantii infection. Plants were infected or mock treated, leaves were harvested $24 \mathrm{~h}$ after treatment. Transcript levels were monitored by qRT-PCR and normalized against the transcripts of the reference genes APT and Clathrin. Bars represent standard deviation. $\mathrm{N}=4$. Experiments were performed three times with similar results. Different letters in the graph indicate statistical significance between treatments (one-way ANOVA with Tuckey's test; $\mathrm{P}<0.05$ ). NI: Non- inoculated, Mock: treated with $\mathrm{MgSO}_{4}$.

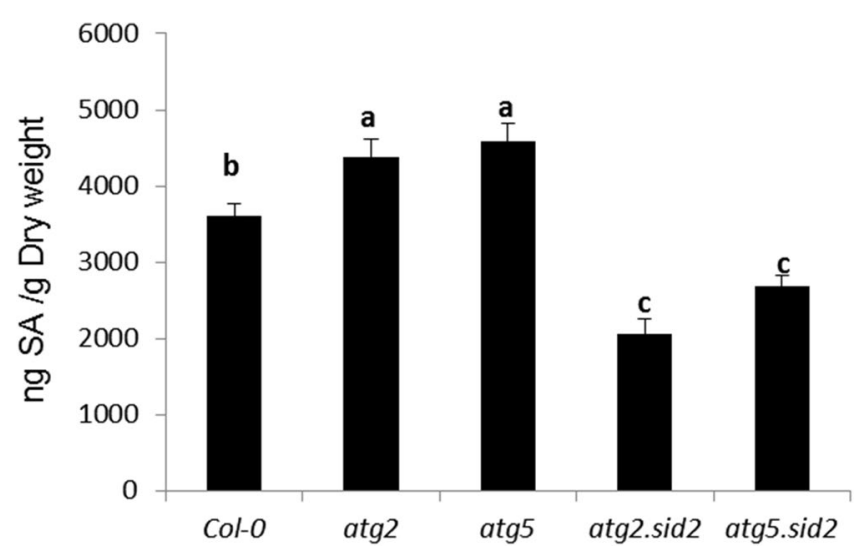

Figure 5. Salicylic acid content in atg mutants: Six week old plants of indicated genotypes were harvested then SA content was monitored as indicated in Materials and Methods. Bars represent standard deviation. $\mathrm{N}=10$ to 12. Experiments were performed three times with similar results. Different letters in the graph indicate statistical significance between treatments (one-way ANOVA with Tuckey's test; $P<0.05$ ). 
mutants in Arabidopsis ${ }^{33,34}$ that is related to leaf yellowing phenotypes and necrotic spots. It might be noticed that atg 2 mutant phenotypes are stronger than those of atg 5 mutant. Our findings showing different behaviors of two autophagy mutants are not surprising with regard to the literature. Indeed, it appears from the literature that the susceptibility of atg mutants to different pathogens is not always similarly affected compared to the WT plants. For instance, although we found that in response to D. dadantii, the atg2 mutant showed enhanced susceptibility, the susceptibility of the same atg2 mutant to the necrotrophic fungus Botrytis cinerea has not been found affected ${ }^{65}$. In $\operatorname{atg} 7$ and $\operatorname{atg} 12$ Arabidopsis mutants, the susceptibility to the necrotrophic fungus Sclerotinia sclerotiorum was shown unaffected ${ }^{66}$. All these reports indicate that although a general trend observed in autophagy mutants is the susceptibility to necrotrophic pathogens, some mutants are likely not affected.

A common trend in necrotrophy is cell wall degrading enzyme production by pathogens. Cell wall homeostasis is tightly linked to membrane stability which could be in part under the control of autophagy ${ }^{29,67,68}$. The mechanisms explaining the higher susceptibility of some atg mutants to necrotrophic pathogens remained unknown $^{44,48}$. One hypothesis is that as a pro-survival mechanism, autophagy hampers the growth and development of necrotrophs which proliferate preferentially on dead cells. In this situation, autophagy would ensure the clearance of degraded cellular components thus protecting plant tissues from activating stress responses such as the SA signaling pathway ${ }^{44}$. Another hypothesis is that the hormonal balance which is disturbed in atg mutants favors necrotrophs. Both hypotheses are not mutually exclusive and can depend upon the the pathosystem considered. In the interaction between Arabidopsis and D. dadantii, we demonstrate that the enhanced susceptibility of the atg2 mutant relies on SA signaling Our data also show that the senescing state of the plants correlates with disease development since $S A G 12$ expression and chlorotic phenotype were enhanced in atg2 plants and lost in the atg2.sid 2 mutant. It is however intriguing that there is no correlation between SA content and PR1 expression level in $\operatorname{atg} 2$ and $\operatorname{atg} 5$ mutants. One interpretation is that senescence and SA form an amplification loop in atg 2 and that both are required to trigger high susceptibility ${ }^{34}$. Such amplification loop between senescence and SA was indeed recently described ${ }^{69,70}$.

We showed that in atg2 higher SA content and signaling coincided with low JA signaling thus explaining increased susceptibility, as JA signaling has been shown to contribute to Arabidopsis tolerance to D. dadantii $i^{50,57}$. The disease phenotype of the atg5 mutant, which is similar to that of the WT plants, may be explained by the fact that atg 5 is senescing later than atg2 mutant. This may be due to a differentially active senescence and immunity molecular machinery in each atg mutant. For instance, transcript levels of genes encoding transcription factors related to immunity and senescence (WRKY, ERF and JAZ) are different in an atg 5 compared to an atg 9 mutant ${ }^{54}$. Interestingly, ATG9 interacts with ATG2 in the phagophore expansion process ${ }^{23}$ suggesting that $\operatorname{atg} 9$ and $\operatorname{atg} 2$ mutants could harbor the similar immunity and senescence defects that differ from those of the atg5 mutant. In addition to the differential expression of transcription factor encoding genes in different atg mutants, it is possible that different proteolytic activities reside in each mutant. Indeed, it was recently shown that autophagy deficiency in the $\operatorname{atg} 5$ mutant resulted in an alteration of cellular proteolytic activities ${ }^{67}$. The actors of immunity including transcription factors may be targeted by proteolysis which ensures fine tuning of adequate defense responses $^{71,72}$. It would be interesting to compare the proteolytic activities in atg 2 and atg 5 mutants to investigate the lifetime of key immunity and senescence related transcription factors.

Altogether, we provide here the evidence that SA plays a pivotal role in the enhanced susceptibility of atg2 mutant to $D$. dadantii through the repression of JA-dependent defenses, and that autophagy degradation function is not directly involved in plant tolerance to $D$. dadantii. While our study argues against the involvement of plant autophagy in the tolerance to this type of aggressors, it confirms the importance of leaf senescence status in the susceptibility of plants and points to the hormonal balance as a key player in this process. In addition, overexpression of ATG8 proteins, that had been shown to provide positive effects on plant tolerance to many stresses such as drought in several plant species (Chen et al. ${ }^{73}$ for a review), significantly increased the tolerance of our Arabidopsis plants to D. dadantii infection.

Received: 3 September 2020; Accepted: 8 January 2021

Published online: 11 February 2021

\section{References}

1. Klessig, D. F., Choi, H. W. \& Dempsey, D. A. Systemic acquired resistance and salicylic acid: Past, present, and future. Mol. PlantMicrobe Interact. 31, 871-888 (2018).

2. Piasecka, A., Jedrzejczak-Rey, N. \& Bednarek, P. Secondary metabolites in plant innate immunity: Conserved function of divergent chemicals. New Phytol. 206, 948-964 (2015).

3. Wang, W., Feng, B., Zhou, J. M. \& Tang, D. Plant immune signaling: Advancing on two frontiers. J. Integr. Plant Biol. 62, 2-24 (2020).

4. Jones, J. D. G. \& Dangl, J. L. The plant immune system. Nature 444, 323-329 (2006).

5. Dangl, J. L., Horvath, D. M. \& Staskawicz, B. J. Pivoting the plant immune system from dissection to deployment. Science 341, 746-751 (2013).

6. Wirthmueller, L., Maqbool, A. \& Banfield, M. J. On the front line: Structural insights into plant-pathogen interactions. Nat. Rev. Microbiol. 11, 761-776 (2013).

7. Dodds, P. N. \& Rathjen, J. P. Plant immunity: Towards an integrated view of plant pathogen interactions. Nat. Rev. Genet. 11, 539-548 (2010).

8. Macho, A. P. \& Zipfel, C. Plant PRRs and the activation of innate immune signaling. Mol. Cell 54, 263-272 (2014).

9. Alhoraibi, H., Bigeard, J., Rayapuram, N., Colcombet, J. \& Hirt, H. Plant immunity: The MTI-ETI model and beyond. Curr. Issues Mol. Biol. 30, 39-58 (2019).

10. Robert-Seilaniantz, A., Grant, M. \& Jones, J. D. G. Hormone crosstalk in plant disease and defense: More than just jasmonatesalicylate antagonism. Annu. Rev. Phytopathol. 49, 317-343 (2011). 
11. Pieterse, C. M. J., Van der Does, D., Zamioudis, C., Leon-Reyes, A. \& Van Wees, S. C. M. Hormonal modulation of plant immunity. Annu. Rev. Cell Dev. Biol. 28, 489-521 (2011).

12. Vos, I. A., Moritz, L., Pieterse, C. M. J. \& Van Wees, S. C. M. Impact of hormonal crosstalk on plant resistance and fitness under multi-attacker conditions. Front. Plant Sci. 6, 639 (2015).

13. Verma, V., Ravindran, P. \& Kumar, P. P. Plant hormone-mediated regulation of stress responses. BMC Plant Biol. 16, 86 (2016).

14. Glazebrook, J. Contrasting mechanisms of defense against biotrophic and necrotrophic pathogens. Annu. Rev. Phytopathol. 43, 205-227 (2005).

15. Andolfo, G. \& Ercolano, M. R. Plant innate immunity multicomponent model. Front. Plant Sci. 6, 987 (2015).

16. Thaler, J. S., Humphrey, P. T. \& Whiteman, N. K. Evolution of jasmonate and salicylate signal crosstalk. Trends Plant Sci. 5, 260-270 (2012).

17. Caarls, L. et al. Assessing the role of ETHYLENE RESPONSE FACTOR transcriptional repressors in salicylic acid-mediated suppression of jasmonic acid-responsive genes. Plant Cell Physiol. 58, 266-278 (2017).

18. Koornneef, A. \& Pieterse, C. M. J. Cross talk in defense signaling. Plant Physiol. 146, 839-844 (2008).

19. Leon-Reyes, A. et al. Salicylate-mediated suppression of jasmonate-responsive gene expression in Arabidopsis is targeted downstream of the jasmonate biosynthesis pathway. Planta 232, 1423-1432 (2010).

20. Van der Does, D. et al. Salicylic acid suppresses jasmonic acid signaling downstream of SCF COI1-JAZ by targeting GCC promoter motifs via transcription factor ORA59. Plant Cell. 5, 744-761 (2013).

21. Mur, L. A. J., Kenton, P., Atzorn, R., Miersch, O. \& Wasternack, C. The outcomes of concentration-specific interactions between salicylate and jasmonate signaling include synergy, antagonism, and oxidative stress leading to cell death. Plant Physiol. 140, 249-262 (2006).

22. Feng, Y., He, D., Yao, Z. \& Klionsky, D. J. The machinery of macroautophagy. Cell Res. 24, 24-41 (2014).

23. Avila-Ospina, L., Moison, M., Yoshimoto, K. \& Masclaux-Daubresse, C. Autophagy, plant senescence, and nutrient recycling. J. Exp. Bot. 65, 3799-3811 (2014).

24. Masclaux-Daubresse, C., Chen, Q. \& Havé, M. Regulation of nutrient recycling via autophagy. Curr. Opin. Plant Biol. 39, 8-17 (2017).

25. Dupont, N. et al. Autophagy-based unconventional secretory pathway for extracellular delivery of IL-1 $\beta$. EMBO J. 30, 4701-4711 (2011).

26. Cavalli, G. \& Cenci, S. Autophagy and protein secretion. J. Mol. Biol. 432, 2525-2545 (2020).

27. Cui, Y. et al. Biogenesis of plant prevacuolar multivesicular bodies. Mol. Plant 9, 774-786 (2016).

28. Soto-Burgos, J., Zhuang, X., Jiang, L. \& Bassham, D. C. Dynamics of autophagosome formation. Plant Physiol. 176, 219-229 (2018).

29. Wun, C. L., Quan, Y. \& Zhuang, X. Recent advances in membrane shaping for plant autophagosome biogenesis. Front. Plant Sci. $11,565(2020)$.

30. Tsukada, M. \& Ohsumi, Y. Isolation and characterization of autophagy-defective mutants of Saccharomyces cerevisiae. FEBS Lett. 33, 169-174 (1993).

31. Yang, X. \& Bassham, D. C. New insight into the mechanism and function of autophagy in plant cells. Int. Rev. Cell Mol. Biol. 320, $1-40(2015)$.

32. Doelling, J. H., Walker, J. M., Friedman, E. M., Thompson, A. R. \& Vierstra, R. D. The APG8/12-activating enzyme APG7 is required for proper nutrient recycling and senescence in Arabidopsis thaliana. J. Biol. Chem. 277, 33105-33114 (2002).

33. Thompson, A. R., Doelling, J. H., Suttangkakul, A. \& Vierstra, R. D. Autophagic nutrient recycling in Arabidopsis directed by the ATG8 and ATG12 conjugation pathways. Plant Physiol. 138, 2097-2110 (2005).

34. Yoshimoto, K. et al. Autophagy negatively regulates cell death by controlling NPR1-dependent salicylic acid signaling during senescence and the innate immune response in arabidopsis. Plant Cell 21, 2914-2927 (2009).

35. Hanaoka, H. et al. Leaf senescence and starvation-induced chlorosis are accelerated by the disruption of an Arabidopsis autophagy gene. Plant Physiol. 129, 1181-1193 (2002).

36. Deprost, D. et al. The Arabidopsis TOR kinase links plant growth, yield, stress resistance and mRNA translation. EMBO Rep. 8, 864-870 (2007).

37. Liu, Y. \& Bassham, D. C. TOR is a negative regulator of autophagy in Arabidopsis thaliana. PLoS ONE 5, el1883 (2010).

38. Pu, Y., Luo, X. \& Bassham, D. C. Tor-dependent and -independent pathways regulate autophagy in Arabidopsis thaliana. Front. Plant Sci. 8, 1204 (2017).

39. Seay, M., Patel, S. \& Dinesh-Kumar, S. P. Autophagy and plant innate immunity. Cell. Microbiol. 8, 899-906 (2006).

40. Yang, M., Bu, F., Huang, W. \& Chen, L. Multiple regulatory levels shape autophagy activity in plants. Front. Plant Sci. 10, 532 (2019).

41. Leary, A. Y., Savage, Z., Tumtas, Y. \& Bozkurt, T. O. Contrasting and emerging roles of autophagy in plant immunity. Curr. Opin. Plant Biol. 52, 46-53 (2019).

42. Leary, A. Y. et al. Modulation of plant autophagy during pathogen attack. J. Exp. Bot. 69, 1325-1333 (2018).

43. Liao, C. Y. \& Bassham, D. C. Combating stress: The interplay between hormone signaling and autophagy in plants. J. Exp. Bot. 71, $1723-1733(2020)$.

44. Zeng, H.Y., Zheng. P., Wang, L.Y., Bao, H.N., Sahu, S.K. \& Yao, N. Autophagy in plant immunity BT-autophagy regulation of innate immunity. In (ed. Cui, J.) 23-41 (Springer Singapore, 2019).

45. Hofius, D. et al. Autophagic components contribute to hypersensitive cell death in Arabidopsis. Cell 137, 773-783 (2009).

46. Lenz, H. D. et al. Autophagy differentially controls plant basal immunity to biotrophic and necrotrophic pathogens. Plant J. 66, 818-830 (2011).

47. Lai, Z., Wang, F., Zheng, Z., Fan, B. \& Chen, Z. A critical role of autophagy in plant resistance to necrotrophic fungal pathogens. Plant J. 66, 953-968 (2011).

48. Zhou, J., Yu, J. Q. \& Chen, Z. The perplexing role of autophagy in plant innate immune responses. Mol. Plant Pathol. 15, 637-645 (2014).

49. Reverchon, S. \& Nasser, W. Dickeya ecology, environment sensing and regulation of virulence programme. Environ. Microbiol. Rep. 5, 622-636 (2013).

50. Fagard, M. et al. Arabidopsis thaliana expresses multiple lines of defense to counterattack Erwinia chrysanthemi. Mol. Plant-Microbe Interact. 20, 794-805 (2007).

51. Kieu, N. P. et al. Iron deficiency affects plant defence responses and confers resistance to Dickeya dadantii and Botrytis cinerea. Mol. Plant Pathol. 13, 816-827 (2012).

52. Aznar, A., Chen, N. W. G., Thomine, S. \& Dellagi, A. Immunity to plant pathogens and iron homeostasis. Plant Sci. 240, 90-97 (2015).

53. Taurino, M. et al. Jasmonate-dependent modifications of the pectin matrix during potato development function as a defense mechanism targeted by Dickeya dadantii virulence factors. Plant J. 77, 418-429 (2014).

54. Masclaux-Daubresse, C. et al. Stitching together the multiple dimensions of autophagy using metabolomics and transcriptomics reveals impacts on metabolism, development, and plant responses to the environment in Arabidopsis. Plant Cell 26, 1857-1877 (2014).

55. Chen, Q. et al. Overexpression of ATG8 in Arabidopsis stimulates autophagic activity and increases nitrogen remobilization efficiency and grain filling. Plant Cell Physiol. 60, 343-352 (2019). 
56. Rigault, M. et al. Quantitative methods to assess differential susceptibility of Arabidopsis thaliana natural accessions to Dickeya dadantii. Front. Plant Sci. 8, 394 (2017).

57. Verly, C. et al. Plant defense stimulator mediated defense activation is affected by nitrate fertilization and developmental stage in Arabidopsis thaliana. Front. Plant Sci. 11, 583 (2020).

58. Aznar, A. et al. Scavenging iron: A novel mechanism of plant immunity activation by microbial siderophores. Plant Physiol. 164, 2167-2183 (2014)

59. Le Roux, C. et al. The hnRNP-Q protein LIF2 participates in the plant immune response. PLoS ONE 9, e99343 (2014).

60. Mansfield, J. et al. Top 10 plant pathogenic bacteria in molecular plant pathology. Mol. Plant Pathol. 13, 614-629 (2012).

61. Wildermuth, M. C., Dewdney, J., Wu, G. \& Ausubel, F. M. Isochorismate synthase is required to synthesize salicylic acid for plant defence. Nature 414, 562-565 (2001).

62. Yoshimoto, K. Plant autophagy puts the brakes on cell death by controlling salicylic acid signaling. Autophagy. 6, 192-193 (2010).

63. Verhage, A., van Wees, S. C. M. \& Pieterse, C. M. J. Plant immunity: It's the hormones talking, but what do they say?. Plant Physiol. 154, 536-540 (2010)

64. Üstün, S. et al. Bacteria exploit autophagy for proteasome degradation and enhanced virulence in plants. Plant Cell 30, 668-685 (2018).

65. Wang, Y., Nishimura, M. T., Zhao, T. \& Tang, D. ATG2, an autophagy-related protein, negatively affects powdery mildew resistance and mildew-induced cell death in Arabidopsis. Plant J. 68, 74-87 (2011).

66. Kabbage, M., Williams, B. \& Dickman, M. B. Cell death control: The interplay of apoptosis and autophagy in the pathogenicity of Sclerotinia sclerotiorum. PLoS Pathog. 9, e1003287 (2013).

67. Havé, M. et al. Proteomic and lipidomic analyses of the Arabidopsis atg5 autophagy mutant reveal major changes in endoplasmic reticulum and peroxisome metabolisms and in lipid composition. New Phytol. 223, 1461-1477 (2019).

68. Zeng, Y., Li, B., Lin, Y. \& Jiang, L. The interplay between endomembranes and autophagy in plants. Curr. Opin. Plant Biol. 2, 14-22 (2019).

69. Guo, P. et al. A tripartite amplification loop involving the transcription factor WRKY75, salicylic acid, and reactive oxygen species accelerates leaf senescence. Plant Cell 29, 2854-2870 (2017).

70. Woo, H. R., Kim, H. J., Lim, P. O. \& Nam, H. G. Leaf senescence: Systems and dynamics aspects. Annu. Rev. Plant Biol. 70, 347-376 (2019).

71. Copeland, C. \& Li, X. Chapter two-regulation of plant immunity by the proteasome. In International Review of Cell and Molecular Biology (ed. Galluzzi, L. B. T.-I. R. of C. and M. B.) Vol. 343, 37-63 (Academic Press, 2019).

72. Caarls, L., Pieterse, C. M. J. \& Van Wees, S. C. M. How salicylic acid takes transcriptional control over jasmonic acid signaling. Front. Plant Sci. 6, 170 (2015).

73. Chen, Q. et al. Autophagy and nutrients management in plants. Cells 8, 1426 (2019).

74. Zhou, J. et al. NBR1-mediated selective autophagy targets insoluble ubiquitinated protein aggregates in plant stress responses. PLoS Genet. 9, e1003196 (2013).

\title{
Acknowledgements
}

Authors thanks Dr. Kohki Yoshimoto (Meiji University, Japan) for mutant seeds. We thank Hervé Ferry for taking care of plants. This work has benefited from the support of IJPB's Plant Observatory technological platforms. The IJPB benefits from the support of Saclay Plant Sciences-SPS (ANR-17-EUR-0007).

\section{Author contributions}

M.R., S.C., A.D. performed experiments. A.D. and C.M.D. designed the work and wrote the manuscript.

\section{Competing interests}

The authors declare no competing interests.

\section{Additional information}

Supplementary Information The online version contains supplementary material available at https://doi. org/10.1038/s41598-021-83067-6.

Correspondence and requests for materials should be addressed to A.D.

Reprints and permissions information is available at www.nature.com/reprints.

Publisher's note Springer Nature remains neutral with regard to jurisdictional claims in published maps and institutional affiliations.

\begin{abstract}
Open Access This article is licensed under a Creative Commons Attribution 4.0 International License, which permits use, sharing, adaptation, distribution and reproduction in any medium or format, as long as you give appropriate credit to the original author(s) and the source, provide a link to the Creative Commons licence, and indicate if changes were made. The images or other third party material in this article are included in the article's Creative Commons licence, unless indicated otherwise in a credit line to the material. If material is not included in the article's Creative Commons licence and your intended use is not permitted by statutory regulation or exceeds the permitted use, you will need to obtain permission directly from the copyright holder. To view a copy of this licence, visit http://creativecommons.org/licenses/by/4.0/.
\end{abstract}

(C) The Author(s) 2021 